Publ. RIMS, Kyoto Univ.

Vol. 6 (1970), 193-200

\title{
Finiteness of the Number of Discrete Eigenvalues of the Schrödinger Operator for a Three Particle System $\mathbb{I I}^{*}$
}

By

Jun UсHгуAмA**

\section{\$. Introduction}

The presert facer is the co tinuation of [2] with the same title. In the previous papers $[1 \mid[2]$, we nave studied the Schrödinger operator of the form

$$
H=-\Delta_{1}-\Delta_{2}-\frac{Z_{1}}{r_{1}}-\frac{Z_{2}}{r_{2}}+\frac{Z_{3}}{\left|r_{1}-r_{2}\right|}
$$

where $Z_{1} \geq Z_{2}$ and $Z_{3}$ are positive constants. There ve have shown the results:

i) If $Z_{2}>Z_{3}, H$ has an infinite number of discrete eigenvalues in $\left(-\infty,-Z_{1}^{2} / 4\right)$.

ii) If $Z_{1}, Z_{2}<Z_{3}, H$ has at most a finite number of discrete eigenvalues in $\left(-\infty,-Z_{1}^{2} / 4\right)$.

In this article we shall study the case $Z_{1} \geq Z_{3}>Z_{2}$. In this case, the conditions in [1] or [2] are not satisfied, but we have the same results as (ii) by modifying slightly the proof of Theorem 1 in [2].

The theorems proved in this parer assert that the number of discrete eigenvalues of the operator $H$ of the form

$$
H=-\Lambda_{1}-\Lambda_{2}+q_{1}\left(\boldsymbol{r}_{1}\right)+q_{2}\left(\boldsymbol{r}_{2}\right)+P\left(\boldsymbol{r}_{1}, \mathbb{r}_{2}\right)
$$

Received January 27. 1970.

Communicated by S. Matsuura.

* This research was partly supported by the Sakkokai Foundation.

** Mathematical Institute, Kyoto University of Industrial Arts and Textile Fibres. 
depends essentially on the behavior of $q_{2}\left(\boldsymbol{r}_{2}\right)$ in the region $r_{2} \geq R$, and $P\left(\boldsymbol{r}_{1}, \boldsymbol{r}_{2}\right)$ in $\left|\boldsymbol{r}_{1}-\boldsymbol{r}_{2}\right|>R$, if

$$
\mu_{1}<\mu_{2},
$$

where

$$
\begin{array}{ll}
\mu_{i}=\inf _{\varphi \in \mathscr{D}_{L^{2}}^{2}\left(R^{3}\right)} \frac{\left(H_{i} \varphi, \varphi\right)_{R^{3}}}{\|\varphi\|^{2}} & (i=1,2), \\
H_{i}=-\Delta_{i}+q_{i}\left(\boldsymbol{r}_{i}\right) & (i=1,2) .
\end{array}
$$

On the other hand the condition (4.3) depends on the behavior of $q_{1}\left(r_{1}\right)$ and $q_{2}\left(r_{2}\right)$ not only at infinity, but also in the whole space $R^{3}$. Then the structure of the spectrum of the operator of the form (4.2) is complicated.

Since the proofs of the theorems are essentially the same as the one applied in [1] and [2], we shall only sketch the outline. For the convenience, we shall use the same notation as the one introduced in $[2]$.

\section{§. Some Theorems and Proofs}

Let $H$ of the form (4.2) satisfy the conditions:

$$
q_{i}\left(\mathbb{r}_{i}\right) \in L_{l o c}^{2}\left(R^{3}\right) \quad(i=1,2) \quad \text { and } \quad P\left(\mathbb{R}_{1}, \mathbb{r}_{2}\right) \in Q_{\alpha}\left(R^{6}\right)
$$

(for some $\alpha>0$ ) are real-valued functions,

$$
q_{i}\left(\boldsymbol{r}_{i}\right)(i=1,2) \text { converge uniformly to zero as } r_{i} \rightarrow \infty \text {, }
$$

$$
P\left(r_{1}, r_{2}\right) \geq 0 \text { in } R^{6}
$$

(5.4) $\quad P\left(\mathbb{r}_{1}, \boldsymbol{r}_{2}\right)$ converges uniformly to zero as $r_{1} \rightarrow \infty$

whenever $\boldsymbol{\nabla}_{2}$ is fixed, and as $\boldsymbol{r}_{2} \rightarrow \infty$ whenever $\boldsymbol{r}_{1}$ is fixed (see (2.2)(2.5) in [2]). Then it is known that

i) if the domain $D(H)$ of $H$ is $\mathscr{D}_{L^{2}}^{5}\left(R^{6}\right), H$ is a lower semi-bounded selfadjoint operator in $L^{2}\left(R^{6}\right)$,

ii) if $\mu_{1} \leq \mu_{2}$, where $\mu_{i}(i=1,2)$ are defined by $(4.3)$ and (4. 4), $\sigma_{e}(H)=\left[\mu_{1}, \infty\right)$ (see Theorem 1 in $\left.\lceil 2\rceil\right)$.

Moreover we remark the fact that if we assume conditions (5.1) and (5.2), and if $D\left(H_{\imath}\right)=\mathscr{D}_{L^{2}}^{1}\left(R^{3}\right)$, then $H_{i}(i=1,2)$ are a lower semi- 
bounded selfadjoint operator in $L^{2}\left(R^{3}\right), \sigma_{e}\left(H_{i}\right)=[0, \infty)$ and $\mu_{i} \leq 0$. Now we have

Theorem 3. If we assume for the operator $H$ of the form (4.2) the conditions (4.3), (5.1)-(5.4) and

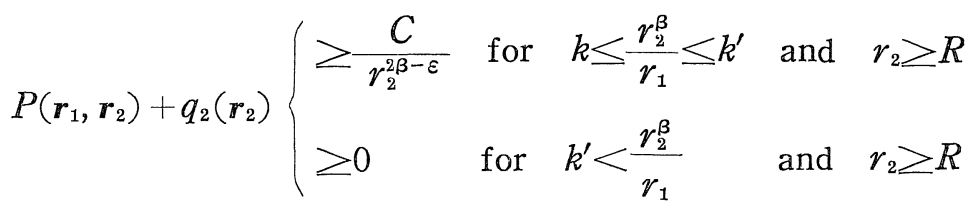

for some constants $k, k^{\prime}\left(1<k<k^{\prime}<+\infty\right), \beta(0<\beta \leq 1), \varepsilon>0, R>0$ and $C>0$, then $H$ has at most a finite number of discreie eigenvalues in $\left(-\infty, \mu_{1}\right)$.

Proof. Let $g(t)$ be a function having the following properties: $g(t) \in C^{\infty}(0, \infty), g(t) \equiv 1$ for $t \geq k^{\prime}, g(i) \equiv 0$ for $0<t<k$ and $0 \leq g(t)$ $\leq 1$ for $0<t<+\infty$. By the conditions (4.3) and (5.2), we can choose $R>1$ large enough to satisfy the following inequalities:

$$
\begin{gathered}
q_{1}\left(r_{1}\right)>\frac{\mu_{1}-\mu_{2}}{3} \text { for } r_{1}>\frac{R^{\beta}}{k^{\prime}} \\
q_{2}\left(r_{2}\right)>\frac{\mu_{1}-\mu_{2}}{3} \text { for } r_{2}>\frac{R^{\beta}}{k^{\prime}}, \\
t^{4} g(t) g^{\prime \prime}(t)+C R^{\varepsilon} \geq 0 \text { for } k \leq t \leq k^{\prime}, \\
\frac{\mu_{2}-\mu_{1}}{3}-\frac{1}{R^{2 \beta}}\left|g(t) g^{\prime \prime}(i) t^{4}\right| \geq 0 \text { for } k \leq t \leq k^{\prime} .
\end{gathered}
$$

Then we define domains $\left\{Q_{i}\right\}_{i=1, \cdots, 4}$ in the same way as in [2], namely

$$
\begin{aligned}
& \Omega_{1}=\left\{r_{1}<R \text { and } r_{2}<R\right\}, \quad \Omega_{2}=\left\{r_{1} \geq R \text { and } r_{2} \leq \frac{r_{1}^{\beta}}{k}\right\}, \\
& \Omega_{3}=\left\{r_{2} \geq R \text { and } r_{1} \leq \frac{r_{2}^{\beta}}{k}\right\} \quad \text { and } \quad \Omega_{4}=R^{6}-\bigcup_{i=1}^{3} \Omega_{i},
\end{aligned}
$$

and for $\psi \in D(H)=D_{L^{2}}^{2}\left(\mathbb{R}^{6}\right)$

$$
\begin{aligned}
L[\psi] & \equiv(H \psi r, \psi r)_{R^{k}}=\sum_{i=1}^{4}\left\{\left\|\left|\nabla_{1} \psi r i\left\|_{l_{2}}^{2}+\right\|\right| \nabla_{2} \psi_{r} \mid\right\|_{t_{2}}^{2}\right. \\
& \left.+\left(q_{1} \psi r, \psi\right)_{\Omega_{2}}+\left(q_{2} \psi r, \psi r\right)_{\Omega_{t}}+(p \psi r, \psi)_{\Omega_{3}}\right\} \equiv \sum_{i=1}^{4} L_{t}[\psi r] .
\end{aligned}
$$


Then we have in the same way as in [2] (see Lemma 1, Lemma 3 and Lemma 4 in $[2]$ )

\section{Lemma 7.}

(i) For any $\psi \in D(H), L_{3}[\psi] \geq \mu_{1}\|\psi\|_{i_{3}}^{2}$.

(ii) For any $\psi_{r} \in D(H), L_{4}[\psi] \geq \mu_{1}\|\psi\|_{\Omega_{4}}^{2}$.

(iii) There exists some finite dimensional subspace $\mathfrak{M}$ in $L^{2}\left(R^{6}\right)$ such that for any $\psi_{\in} \in D(H) \cap \mathfrak{M}^{\perp}, L_{1}[\psi] \geq \mu_{1}\|\psi\|_{s_{1}}^{2}$.

In fact we have orly to take ir.to account for (i) that we have for any $\varphi \in \mathscr{D}_{L^{2}}^{2}\left(R^{3}\right) \quad\left(H_{1} \varphi, \varphi\right)_{\mathbb{R}^{3}} \geq \mu_{1}\|\varphi\|_{R^{3}}^{2}$, and for (ii) $\frac{\mu_{1}-\mu_{2}}{3}>\frac{\mu_{1}}{2}$ because of $\mu_{1}<\mu_{2} \leq 0$.

Now we shall show by modifying the proof of Lemma 1 in [2].

Lemama 8. For any $\psi \in D(H), L_{2}[\psi] \geq \mu_{1}\|\psi\|_{-2}^{2}$.

Proof. Malirg use of the relation $\left(H_{2} \varphi, \varphi\right)_{R^{3}} \geq \mu_{2}\left\|_{\varphi}\right\|_{R^{3}}^{2}$ for any $\varphi \in \mathscr{L}_{L^{2}}^{2}\left(R^{3}\right)$, we have in the same way as the proof of Lemma 1 in [2].

$$
\begin{aligned}
L_{2}[\psi] \geq & \int_{-_{2}}\left\{\mu_{2} g\left(\frac{r_{1}^{\beta}}{r_{2}}\right)^{2}+g\left(\frac{r_{1}^{\beta}}{r_{2}}\right) g^{\prime \prime}\left(\frac{r_{1}^{\beta}}{r_{2}}\right) \cdot \frac{r_{1}^{2 \beta}}{r_{2}^{4}}\right. \\
& \left.+q_{2}\left(r_{2}\right)\left(1-g\left(\frac{r_{1}^{\beta}}{r_{2}}\right)^{2}\right)+P+q_{1}\right\}|\psi|^{2} d x
\end{aligned}
$$

for any $\psi \in D(H)$. Let $\frac{r_{1}^{\beta}}{r_{2}}=t$, and we have by (5.6)-(5.8)

$$
\begin{gathered}
\left(1-g(i)^{2}\right)\left(-\mu_{2}+q_{2}\left(\mathbb{r}_{2}\right)\right)+\left(\mu_{2}-\mu_{1}\right)+\frac{1}{r_{1}^{2 \beta}} g(t) g^{\prime \prime}(t) t^{4} \\
+q_{1}\left(\mathbb{r}_{1}\right)+P\left(g_{1}, \boldsymbol{r}_{2}\right) \geq 0
\end{gathered}
$$

frr $t \geq k$ and $r_{1} \geq R$. in fact $g(t) \equiv 1$ and $g^{\prime \prime}(t) \equiv 0$ for $t \geq k^{\prime}$, and $(1-$ $\left.g(t)^{2}\right)\left(-\mu_{2}+q_{2}\left(\mathbb{r}_{2}\right)\right) \geq \frac{\mu_{1}-\mu_{2}}{3}$ for $k \leq t<k^{\prime}$ i. e. $r_{2}>\frac{r_{1}^{\beta}}{k^{\prime}}>\frac{R^{\beta}}{k^{\prime}}, P\left(\mathbb{r}_{1}\right.$, $\left.\mathbb{P}_{2}\right) \geq 0$, and $q_{1}\left(\mathbb{R}_{1}\right) \geq \frac{\mu_{1}-\mu_{2}}{3}$ for $\gamma_{1}>R>\frac{R^{3}}{k^{\prime}}$. Therefore for any $\psi \in$ $D(H)$, we bave $L_{2}\lfloor\psi] \geq \mu_{1}\left\|\psi_{\psi}\right\|_{c_{2}}^{2}$ by $(5.10)$ and $(5.11)$.

Making use of Lemma 7 and Lemma 3 in the same way as applied in the proof of Theorem 1 in [2], we have the assertion of Theorem 3. 
Remark 6. The operator of the form (4.1) has at most a finite number of discrete eigenvalues in $\left(-\infty, \mu_{1}\right)$, if $Z_{1} \geq Z_{3}>Z_{2}$. In fact $\mu_{1}=-\frac{Z_{1}^{2}}{4}<\mu_{2}=-\frac{Z_{2}^{2}}{4}$ and the condition (5.5) is satisfied (see, Remark 1 in [2]).

If $q_{2}\left(\boldsymbol{r}_{2}\right)$ tends to zero more rapidly than the conditions given in Remark 1 in [2] which satisfies (5.5), we have only to assume (5.3) in place of (5.3) and (5.5) as for $P\left(\boldsymbol{r}_{1}, \boldsymbol{r}_{2}\right)$. Namely we have

Theorem 4. If we assume (4.3), (5.1)-(5.4) and the condition

$$
q_{2}\left(\boldsymbol{r}_{2}\right) \geq-\frac{1}{4} \frac{1}{r_{2}^{2}} \text { for } r_{2} \geq R
$$

the Schrödinger operator $H$ of the form (4.2) has at most a finite number of discrete eigenvalues in $\left(-\infty, \mu_{1}\right)$.

Proof. Let

$$
T=-\Delta_{1}-\Delta_{2}+q_{1}\left(\boldsymbol{r}_{1}\right)+q_{2}\left(\boldsymbol{r}_{2}\right) .
$$

If $D(T)=D(H)=\mathscr{L}_{L^{2}}^{2}\left(R^{6}\right), T$ is a selfadjoint operator in $L^{2}\left(R^{6}\right)$ and $\sigma_{e}(T)=\sigma_{\epsilon}(H)=\left[\mu_{1}, \infty\right)$. By (5.12) $H_{2}$ has at most a finite number of discrete eigenvalues in $(-\infty, 0)$. Let the discrete eigenvalues of $H_{2}$ be $\lambda_{1}^{(2)}=\mu_{2} \leq \lambda_{2}^{(2)} \leq \cdots \leq \lambda_{n}^{(2)}<0 \quad$ ( $m$ is finite), if they exist, and let those of $H_{1}$ be $\lambda_{1}^{(1)}=\mu_{1} \leq \lambda_{a}^{(1)} \leq \cdots \leq \lambda_{n}^{(1)}<0$ ( $n$ may be infinite). Then by the method of the separation of variables, we have, by the method applied to the proof of Lemma 6 in [2],

Lemma 9. If $\lambda$ is an eigenvalue of $T$ smaller than $\mu_{1}$, then $\lambda \in\left\{\lambda_{k}^{(1)}+\lambda_{l}^{(2)}\right\}_{k=1, \cdots, n, l=1, \cdots, m}$.

Taking into consideration that $\left\{\lambda_{k}^{(1)}+\lambda_{l}^{(2)}\right\}_{k=1, \cdots, n, l=1, \cdots, m}$ concentrates at most $\left\{\lambda_{l}^{(2)}\right\}_{l=1, \cdots, m}, T$ has at most a finite number of discrete eigenvalues in $\left(-\infty, \mu_{1}\right)$ by Lemma 9 and the condition (4.3). Let $\mathfrak{N}$ be the finite dimensional subspace in $L^{2}\left(R^{6}\right)$ spanned by the eigenfunctions belonging to the eigenvalues of $T$ in $\left(-\infty, \mu_{1}\right)$. Then by (5.3) and $\sigma_{e}(T)=\left[\mu_{1}, \infty\right)$ we have for any $\psi \in D(H) \cap \mathfrak{N}^{\perp}$,

$$
\left(H \psi r, \psi^{\prime}\right)_{R^{6}} \geq\left(T \psi, \psi^{\prime}\right)_{R^{6}} \geq \mu_{1}\|\psi\|_{R^{6}}^{2},
$$


which asserts that Theorem 2 holds.

In case $\mu_{1} \leq \mu_{2}$, in order to obtain the result that $H$ has at most a finite number of discrete eigenvalues in $\left(-\infty, \mu_{1}\right)$, we must impose some conditions on the behavior of $q_{1}\left(\boldsymbol{r}_{1}\right)$ for $r_{1} \geq R$, and $P\left(\mathbb{r}_{1}, \mathbb{r}_{2}\right)$ for $\left|\boldsymbol{r}_{1}-\boldsymbol{r}_{2}\right| \geq R$ in addition to (5.5) or (5.12) (see, for example, Theorem 1 or Theorem 2 in [2]). Otherwise there exists the case that $H$ has an infinite number of discrete eigenvalues in $\left(-\infty, \mu_{1}\right)$. Namely we have

Theorem 5. If we assume (5.1)-(5.4) and

$$
\begin{gathered}
\mu_{1} \leq \mu_{2}, \\
q_{2}\left(\boldsymbol{r}_{2}\right) \leq-\frac{c}{r_{2}^{\beta}} \quad \text { for } \quad r_{2} \geq R_{0}, \\
0 \leq P\left(r_{1}, r_{2}\right)\left\{\begin{array}{l}
\leq \frac{d R_{1}^{\beta^{\prime}-\beta} R_{2}^{\gamma-\beta}}{\left|r_{1}-r_{2}\right|^{\gamma}} \text { for } \quad\left|r_{1}-r_{2}\right| \leq R_{2}, \\
\leq \frac{d R_{1}^{\beta^{\prime}-\beta}}{\left|\boldsymbol{r}_{1}-\boldsymbol{r}_{2}\right|^{\beta^{\prime}}} \text { for } \quad R_{2} \leq\left|\boldsymbol{r}_{1}-r_{2}\right| \leq R_{1}, \\
\leq \frac{d}{\left|r_{1}-r_{2}\right|^{\beta}} \text { for } \quad R_{1} \leq\left|\mathbb{r}_{1}-r_{2}\right|,
\end{array}\right.
\end{gathered}
$$

for some constants $\beta(0<\beta \leq 2), \gamma(0<\gamma<3 / 2), \beta^{\prime}\left(\max (\beta, \gamma)<\beta^{\prime}<3\right)$, $c>0, d>0, R_{2}\left(0<R_{2}<1\right)$ and sufficiently large $R_{0}>0, R_{1}>0$, and

$$
c-d\left\{\begin{array}{l}
>0 \text { for } \quad 0<\beta<2, \\
>\frac{1}{4} \text { for } \quad \beta=2,
\end{array}\right.
$$

then there exist an infinite number of discrete eigenvalues in $(-\infty$, $\left.\mu_{1}\right)$.

Proof. We can prove the above theorem in a manner similar to [1].

By (5.16) and (5.18) ((5.18) is necessary for $\beta=2), H_{2}$ has an infinite number of discrete eigenvalues in $(-\infty, 0)$. Then taking account of (5.15) and $\sigma_{e}\left(H_{1}\right)=\sigma_{\epsilon}\left(H_{2}\right)=[0, \infty), \mu_{1}$ is a discrete eigenvalue of $H_{1}$. Let a ncrmalized eigenfunction belonging to $\mu_{1}$ be $\varphi_{0}\left(\boldsymbol{r}_{1}\right) \in D\left(\boldsymbol{H}_{1}\right)=$ $\mathscr{L}_{L^{2}}^{2}\left(R^{3}\right)$. Moreover we can choose the function having the following 
property (see Lemma 5 in $[1\rfloor$ ); for $\varepsilon>0$ satisfying the relation $c-$ $d-\frac{1}{4-\varepsilon}>0$ in case $c-d>\frac{1}{4}$

$$
\begin{aligned}
& g_{1}\left(\boldsymbol{r}_{2}\right) \in C_{0}^{\infty}\left(R^{3}\right),\left\|g_{1}\right\|_{R^{3}}=1, g_{1}\left(\boldsymbol{r}_{2}\right) \equiv 0 \text { for } r_{2} \leq R_{0} \\
& \left.\left|\int_{R^{3}} \frac{\left|g_{1}\left(\boldsymbol{r}_{2}\right)\right|^{2}}{r_{2}^{2}} d \boldsymbol{r}_{2} \geq(4-\varepsilon) \int_{R^{3}}\right| \nabla_{1} g_{1}\right|^{2} d \boldsymbol{r}_{2} .
\end{aligned}
$$

Let $g_{\alpha}\left(\boldsymbol{r}_{2}\right)=a^{3 / 2} g_{1}\left(\alpha \boldsymbol{r}_{2}\right)$ and $\psi_{\alpha}(x)=\varphi_{0}\left(\boldsymbol{r}_{1}\right) g_{\alpha}\left(\boldsymbol{r}_{2}\right)$, where $\alpha$ is a positive parameter, we have $\left\|\psi_{\alpha}\right\|_{R^{6}}=1$ and $\psi_{\alpha}(x) \in D(H)=\mathscr{D}_{L^{2}}^{2}\left(R^{6}\right)$. Then taking account of the relation

(5.20) $\lim _{\alpha \rightarrow 0} \int_{R^{6}} \frac{\left|\varphi_{0}\left(\boldsymbol{r}_{1}\right) g_{1}\left(\boldsymbol{r}_{2}\right)\right|^{2}}{\left|\alpha \boldsymbol{r}_{1}-\boldsymbol{r}_{2}\right|^{\overline{0}}} d x=\int_{R^{3}} \frac{\left|g_{1}\right|^{2}}{r_{2}^{S}} d \boldsymbol{r}_{2}$ for any $\delta(0<\delta<3)$, (see Lemma 4 in $\left[1_{\perp}\right)$, there exists some constant $a_{0}^{\prime}\left(0<\alpha_{0}^{\prime}<1\right.$ ) such that for any $a\left(0<a<\alpha_{0}^{\prime}\right)$ we have

$$
\left(H_{\psi} \psi_{\alpha}, \psi r_{\alpha}\right)_{R^{6}} \leq \mu_{1}+M \alpha^{\beta^{\prime}}-\left\{\begin{array}{r}
\frac{1}{2}(c-d) \alpha^{\beta} \int_{R^{3}} \frac{\left|g_{1}\right|}{{ }^{2} \gamma_{2}^{\beta}} d r_{2}+M^{\prime} \alpha^{2} \\
\quad(\text { for } 0<\beta<2) \\
\frac{1}{4}\left(c-d-\frac{1}{4-\varepsilon}\right) \alpha^{2} \int_{R^{3}} \frac{\left|g_{1}\right|^{2}}{r_{2}^{2}} d r_{2} \\
\quad(\text { for } \beta=2),
\end{array}\right.
$$

where $M$ and $M^{\prime}$ are constants independent of $\alpha$ (see (4.9) or (4.9') in $[1])$. Then by (5.18) and (5.21) there exists some constant $\alpha_{0}\left(0<a_{0}<\alpha_{0}^{\prime}\right)$ such that for any $\alpha\left(0<\alpha<\alpha_{0}\right)$ we have

$$
\left(H \psi_{\alpha}, \psi_{\alpha}\right)_{R^{6}}<\mu_{1} .
$$

Now we assume that $H$ has at most a finite number of discrete eigenvalues in $\left(-\infty, \mu_{1}\right)$. Let their number be $p$, and the subspace in $L^{2}\left(R^{6}\right)$ spanned by their eigenfunctions be $\mathfrak{M}$. Then we can choose $\left\{\alpha_{i}\right\}_{i-1, \cdots, p+1}$ such that $0<\alpha_{p+1}<\alpha_{p}<\cdots<\alpha_{1}<\alpha_{0}$ and the support of $g_{a_{2}}\left(r_{2}\right)$ and that of $g_{\alpha_{1}}\left(r_{2}\right)$ are disjoirt in $R^{3}$ for $i \neq j$. Since the dimeission of the subspace spanned by $\left\{\varphi_{0}\left(\boldsymbol{r}_{1}\right) g_{\alpha_{1}}\left(\boldsymbol{r}_{2}\right)\right\}_{i=1, \cdots, p+1}$ is $p+1$, we can choose constants $\left\{c_{\imath}\right\}_{i=1, \cdots, p+1}$ such that $\sum_{i=1}^{p+1} c_{\imath} \psi_{r_{\alpha}}(x) \in \mathfrak{M}^{\perp} \cap D(H)$ and $\sum_{i=1}^{p+1}\left|c_{i}\right|^{2}=1$. Let $f(x)=\sum_{i=1}^{p+1} c_{i} \psi_{\alpha_{i}}(x)$. Then by (5.22) and the condition 
that the supports of $g_{\alpha}$, and $g_{\alpha}$, are disjoint for each $i \neq j$, we have $\|f\|_{R^{6}}=1$ and

$$
(H f, f)_{R^{6}}=\sum_{i=1}^{p+1}\left|c_{i}\right|^{2}\left(H \psi r_{\alpha_{t}}, \psi r_{\alpha_{t}}\right)_{R^{6}}<\mu_{1} \sum_{i=1}^{p+1}\left|c_{i}\right|^{2}=\mu_{1} .
$$

On the other hand by $f \in \mathfrak{M}^{\perp} \cap D(H)$ and $\|f\|_{R^{6}}=1$, we have ( $H f$, $f)_{R^{6}} \geq \mu_{1}$, which contradicts (5.23). Thus the assertion of Theorem 5 is proved.

\section{References}

[1] Uchiyama, J., On the discrete eigenvalues of the many-particle system, Publ. RIMS. Kyoto Univ Ser. A 2 (1966), 117-132.

[2] - - Finiteness of the number of discrete eigenvalues of the Schrödinger operator for a three particle system, Publ. RIMS, Kyoto Univ. 5 (1969), 51-63. 